\title{
Sildenafil can induce the onset of a cluster headache bout
}

\author{
Guan-Yu Lin, MD; Jiunn-Tay Lee, MD; Giia-Sheun Peng, MD, PhD; Fu-Chi Yang, MD
}

Department of Neurology, Tri-Service General Hospital, National Defense Medical Center, Taipei, Taiwan, R.O.C.

Cite as: Can Urol Assoc J 2014;8(5-6):e378-80. http://dx.doi.org/10.5489/cuaj.1736 Published online May 21, 2014.

\section{Abstract}

About $25 \%$ of patients who are prescribed sildenafil, the phosphodiesterase type 5 (PDE-5) inhibitor, for erectile dysfunction (ED) experience headaches. These migraine effects are well-described, including cluster headaches. We report the case of a man who experienced a cluster headache attack following each of 2 sildenafil doses. His symptoms were resolved by adding naproxen to his treatment regimen and changing his ED treatment from $50 \mathrm{mg}$ of sildenafil to $5 \mathrm{mg}$ of vardenafil. To our knowledge, no study has reported cluster headaches triggered by the less commonly used PDE-5 inhibitors, namely vardenafil and tadalafil. Urologists should be cautious in prescribing sildenafil to patients with ED and with a history of cluster headaches. In these patients, they should consider prescribing low-dose vardenafil or tadalafil instead. Failure to recognize sildenafil risks could result in unnecessary headache bouts in patients with a history of cluster headaches.

\section{Introduction}

Sildenafil citrate (Viagra, Pfizer), a selective phosphodiesterase type 5 (PDE-5) inhibitor, is effective for erectile dysfunction (ED). Combined with sexual stimulation, PDE-5 inhibition increases cyclic guanosine monophosphate concentrations, which relaxes the corpus cavernosum smooth muscle, resulting in increased blood flow and intra-cavernosal pressure and facilitating penile erection. ${ }^{1}$

Moreira and colleagues reported the most common adverse events associated with sildenafil were flushing $(30.8 \%)$, headache $(25.4 \%)$, nasal congestion $(18.7 \%)$, and heartburn (10.5\%). ${ }^{1}$ Moreover, significant associations between dosage and adverse event incidence were noted. ${ }^{1}$ Headaches occurring with sildenafil are usually migraine type. ${ }^{2}$ Typically, a cluster headache manifests in bouts and persists from 7 days to several months, during which patients experience recurrent attacks, with remission lasting months or years. ${ }^{3}$

We report the case of a man with a history of cluster headache. He experience a cluster headache bout following sildenafil treatment for ED. This report reminds urologists to consider the risk of this side effect with sildenafil use.

\section{Case report}

A 62-year-old man started experiencing cluster headaches at 40 years of age. He had no history of chronic alcoholic use, cigarette smoking, hypertensive cardiovascular disease, or diabetes. The headaches were characterized by right periorbital and right-sided stabbing pains with a numeric rating scale (NRS) intensity of 10/10 and were occasionally associated with nausea and vomiting. They were accompanied by redness and tearing of the right eye and right naris congestion. The headaches occurred once, and occasionally twice, per day, typically lasting for 1.5 to 2 hours. Typically, he experienced a single 4-month-long bout each year until 2 years ago, when he began taking verapamil (sustained release, $240 \mathrm{mg}$ daily). He reported no further headaches at regular follow-ups.

The patient experienced intermittent mild ED (International Index of Erectile Function [IIEF] score: 24) for a year and we thus prescribed sildenafil $(50 \mathrm{mg}$ ). Thirty minutes after taking his first sildenafil pill, he experienced a typical cluster headache attack characterized by right periorbital and rightsided stabbing pain accompanied by redness and tearing of the right eye and right naris congestion. He described the headache as having an NRS intensity of $8 / 10$ and lasting 30 to 60 minutes, but he did not seek immediate treatment. The next day, he repeated the sildenafil tablet and again experienced a typical cluster headache attack about 30 minutes later. He stopped using sildenafil thereafter. Subsequently, he entered a cluster headache bout period, during which he had 1 or 2 attacks a day, with each attack lasting 30 to 40 minutes. He used sumatriptan tablets repetitively to alleviate the attacks with fairly consistent success. 
He visited our outpatient department a week after the cluster headache episode. His neurological examination findings and brain magnetic resonance imaging (MRI) scan were normal. We prescribed a daily naproxen treatment for long-lasting pain relief in addition to the verapamil he was already taking, and changed his 50-mg sildenafil prescription to on-demand low-dose $(5 \mathrm{mg})$ vardenafil. Within 5 days, he experienced relief from the cluster headache attack. At follow-up appointments, the patient reported significant ED improvement with the low-dose vardenafil and no cluster headache bouts.

\section{Discussion}

A cluster headache is a trigeminal autonomic cephalalgia involving activation of the trigeminal-autonomic reflex, but its exact cause is still being debated. ${ }^{4}$ Functional imaging findings together with the circadian biological changes and neuroendocrine alterations that characterize cluster headache have implicated the hypothalamus, especially the posterior hypothalamus, as a key area in the cluster headache etiology. ${ }^{4}$ Although cluster headache is a primary headache disorder, it has been reported to occur secondarily to uncommon pathologies, such as vascular lesions, multiple sclerosis, and pituitary tumours. Therefore, neuroimaging and neurological evaluations should be considered in all patients with cluster headache-like symptoms. ${ }^{5}$ In our case, we conducted a brain MRI to exclude secondary causes and found no significant abnormal findings.

Headaches are common adverse events among patients receiving 50 to $150-\mathrm{mg}$ sildenafil, with increased doserelated occurrence. ${ }^{1}$ The mechanism underlying sildenafiltriggered headaches has not been determined. Cluster headaches may develop due to the weak inhibitory activity of the drug on PDE-6 and -11, in addition to strong inhibitory effects on PDE-5. Central inhibition of PDE-11 can cause cerebral vasodilatation and migraine attacks. ${ }^{2}$ Four cases of cluster headaches triggered by sildenafil were reported between 1999 and 2006. ${ }^{6-8}$ Common triggers of cluster headache include exogenous histamine, vasodilators (e.g., alcohol, nitroglycerin), and cigarette smoking. ${ }^{9}$ In our case, the patient experienced a cluster headache attack after taking each of two 50-mg sildenafil pills on 2 consecutive days, and a subsequent transition to a cluster headache bout. The attacks were symptomatically similar to his spontaneous cluster headaches, except that each attack was shorter than usual, and the subsequent bout period was shorter than he had experienced before. Given that no known triggering factors (mentioned above) were noted during the period of sildenafil exposure, we strongly suspected a causal relationship between the sildenafil use and the patient's subsequent cluster headache bout.
The Cologne study in $2000^{10}$ showed an overall ED prevalence of $19.2 \%$ in men aged 30 to 80 ; this incidence increases with age. ED is also prevalent in men with regular alcohol consumption $(38 \%)$, arterial hypertension $(30 \%)$, smoking $(30 \%)$, and diabetes mellitus $(20 \%) .{ }^{10}$ The primary goal of ED management is to treat underlying etiologies when possible, since ED is often a symptom of another underlying problem. ${ }^{11}$ However, erectile function cannot be improved significantly with organic risk-factor management alone. ${ }^{12}$

ED treatment includes erection-supporting vacuum pumps, psychological sex therapy, intra-urethral or intracavernosal injections of vasoactive substances (the prostaglandin analogue alprostadil), and oral dopamine receptor agonists (apomorphine); the most effective therapies are PDE-5 inhibitors. ${ }^{13}$ All three PDE-5 inhibitors available on the market (sildenafil, vardenafil, and tadalafil) display good efficacy, with sildenafil having the best efficacy $(84 \%) .^{12}$ These inhibitors are thought to produce adverse secondary effects with similar frequency, severity, and dose dependence, but further studies are required to confirm the incidence of adverse events, especially in relation to the lesser prescribed vardenafil and tadalafil. ${ }^{12}$

Vardenafil can be taken orally before sexual activity. At doses in the range of 5 to $20 \mathrm{mg}$, vardenafil yields significant improvements in penetration and maintenance of erection, as evaluated by IIEF scoring, relative to placebo. ${ }^{14}$ Although the recommended dose of vardenafil in adult patients is $10 \mathrm{mg}$, it can be adjusted to a maximum $20 \mathrm{mg}$ or a minimum $5 \mathrm{mg}$ based on efficacy and tolerability. ${ }^{14}$ In our case, we chose on-demand vardenafil at the minimum effective dose $(5 \mathrm{mg})$ to treat the patient's mild ED to avoid potential dose-dependent adverse effects. With regular follow-up, the patient reported good tolerance and a significant improvement of his ED with low-dose vardenafil.

To our knowledge, no study has reported on cluster headaches triggered by vardenafil and tadalafil. Therefore, patients who experience he6adaches following sildenafil use, particularly cluster headaches, may be better treated with low-dose vardenafil or tadalafil than with a standard sildenafil dose.

\section{Conclusion}

In light of the present case, we suggest that urologists exercise caution in prescribing PDE-5 inhibitors to patients with ED and with a history of cluster headaches. In particular, urologists might consider prescribing low-dose vardenafil or tadalafil. Failure to recognize the potential risk of sildenafilinduced cluster headaches could result in cluster headache bouts that have a major negative influence on quality of life.

Competing interests: Dr. Lin, Dr. Lee, Dr. Peng and Dr. Yang declare no competing financial or personal interests. 
Lin et al.

This paper has been peer-reviewed.

\section{References}

1. Moreira J SG, Brannigan RE, Spitz A, et al. Side-effect profile of sildenafil citrate (Viagra) in clinical practice. Urology 2000;56:474-6. http://dx.doi.org/10.1016/S0090-4295(00)00649-X

2. Ferrari A. Headache: One of the most common and troublesome adverse reactions to drugs. Curr Drug Safety 2006;: :43-58. http://dx.doi.org/10.2174/157488606775252610

3. Headache Classification Committee of the International Headache Society. The International Classification of the Headache Disorders, 2nd edition. Cephalalgia 2004:24:1-160.

4. Leone M, Bussone G. Pathophysiology of trigeminal autonomic cephalalgias. Lancet Neurol 2009;8:75564. http://dx.doi.org/10.1016/S1474-4422(09)70133-4

5. Benoliel R. Trigeminal autonomic cephalgias. Br J Pain 2012;6:106. http://dx.doi. org $/ 10.1177 / 2049463712456355$

6. Stein M. Viagra and cluster headache. Headache 1999;39:58-9.

7. Randolph W, Evans MD. Sildenafil can trigger cluster headaches. Headache 2006;46:173-4. http:// dx.doi.org/10.1111/i.1526-4610.2006.00316_4.x
8. de L Figuerola M, Bruera 0, Leston J, et al. Cluster headache attack due to sildenafil intake. Cephalalgia 2006;26:617-9.

9. Weaver-Agostoni J. Cluster headache. Am Fam Physician 2013;88:122-8.

10. Braun M, Wassmer G, Klotz T, et al. Epideminology of erectile dysfunction: Results of the "Cologne Male Survey." Int I Imp Res 2000;12:305-11.

11. Wespes E, Amar E, Hatzichristou D, et al. Guidelines on Erectile Dysfunction. Europ Urol 2002;41:1-5. http://dx.doi.org/10.1016/S0302-2838(01)00008-2

12. Montorsi F, Salonia A, Zanoni M, et al. Current status of local penile therapy. Int J Imp Res 2002; 14:S7081.

13. Gresser $\mathrm{U}$, Gleiter $\mathrm{CH}$. Erectile dysfunction: Comparison of efficacy and side effects of the PDE-5 inhibitors sildenafil, vardenafil, and tadalafil review of the literature. Eur J Med Res 2002;7:435-46.

14. Keating GM, Scott U. Spotlight on vardenafil in erectile dysfunction. Drugs Aging 2004;21:135-40. http://dx.doi.org/10.2165/00002512-200421020-00005

Correspondence: Dr. Fu-Chi Yang, National Defense Medical Center, No. 325, Section 2, Cheng-Kung Road, Neihu 114, Taipei, Taiwan, R.0.C; fuij-yang@yahoo.com.tw 